\title{
Experiencia en la Creación de una Plataforma Online para Alojar Modelos Tridimensionales de Piezas Anatómicas Reales para ser Compartidas como Recursos Educativos Abiertos (REA)
}

\author{
Experience in the Creation of an Online Platform to Host Three-Dimensional Models \\ of Real Anatomical Pieces to be Shared as Open Educational Resources (OER)
}

\author{
Gonzalo Tiznado-Matzner ${ }^{1}$; Sandra Bucarey-Arriagada ${ }^{1} \&$ Rodrigo Lizama Pérez $^{2}$
}

\begin{abstract}
TIZNADO-MATZNER, G.; BUCAREY-ARRIAGADA, S. \& LIZAMA, P. R. Experiencia en la creación de una plataforma online para alojar modelos tridimensionales de piezas anatómicas reales para ser compartidas como recursos educativos abiertos (REA). Int. J. Morphol., 37(4):1267-1271, 2019.
\end{abstract}

RESUMEN: Por mucho tiempo la enseñanza de la anatomía humana, aparte de disecciones y observaciones de muestras cadavéricas se ha basado en textos y posteriormente complementado con softwares de imágenes bidimensionales (2D) o modelos que imitaban estructuras anatómicas. Con el desarrollo de la tecnología en los últimos años, el modelaje digital tridimensional (3D) ha ido teniendo auge, a lo que la anatomía no ha quedado ajena. A través de la fotogrametría digital se puede lograr este objetivo, ya que es un método que consiste en capturar un gran número de imágenes desde los diferentes ángulos de un objeto, en un ambiente de luz controlado, para después ser ingresadas a un software especializado, que se encarga de agruparlas y generar la información necesaria para que el volumen y profundidades del objeto sean convertidos en una malla de polígonos, para posteriormente ser revestidas con una textura, obteniendo así, un modelo digital 3D. El problema de este método es el tiempo que conlleva tomar las imágenes de buena calidad. Por este motivo que a través de la Dirección de Investigación y Desarrollo de la Universidad Austral de Chile, se adquirió un escáner 3D, equipo que tiene la posibilidad de obtener gran cantidad de imágenes en diferentes ángulos en menor tiempo, y que gracias a su propio software permite crear los modelos digitales 3D. Se han digitalizado 76 muestras anatómicas de nuestro laboratorio de anatomía humana, las cuales fueron subidas a la plataforma web Sketchfab, red social que se especializa en compartir modelos digitales 3D. Posterior a esto se creó una página web abierta que permite organizar los modelos alojados en Sketchfab, según el segmento corporal al cual pertenecen. A esta página puede acceder cualquier persona, desde cualquier lugar que tenga internet, a través de un computador personal, celular inteligente o tablets, convirtiéndolo en un recurso educativo abierto (REA).

PALABRAS CLAVE: Modelos digitales 3D; Muestras Anatómicas; Recurso educativo abierto; Plataforma web.

\section{INTRODUCCIÓN}

Los modelos digitales tridimensionales (3D) han sido una gran revolución en los últimos años, debido a la posibilidad de poder observar una estructura desde todos los ángulos y perspectivas, desde un dispositivo electrónico como computadores personales, celulares inteligentes o tablets. A finales del siglo XX y principios del siglo XXI se ofrecían softwares a altos precios, inaccesibles para personas comunes y corrientes y en muchos casos, también, para las universidades (Hunter, 2000). Estos consistían en estructuras modeladas a través de dibujos computarizados. En la actualidad, el avance de la tecnología, ha permitido que el acceso para desarrollar modelos 3D, sea mucho más factible y eco- nómico (Pérez Gutiérrez \& Uribe Quevedo, 2009). Una de estas técnicas, es la fotogrametría digital, que consiste en la utilización de una cámara fotográfica digital simple o profesional, incluyendo las que vienen en los teléfonos móviles actuales. El procedimiento consiste en capturar un conjunto de fotografías bidimensionales (2D) de la estructura en los diferentes ángulos del objeto a modelar (Petriceks et al., 2018). Éstas son agregadas a un software especializado, que se pueden encontrar en Internet de forma gratuita. Éste se encarga de agrupar el conjunto de imágenes $2 \mathrm{D}$, que brindarán la información necesaria para que el volumen y profundidades del objeto sean representados como una nube de

\footnotetext{
${ }^{1}$ Instituto de Anatomía, Histología y Patología, Facultad de Medicina. Universidad Austral de Chile, Chile.

${ }^{2}$ Departamento de Ciencias Morfológicas, Facultad de Medicina y Ciencia, Universidad San Sebastián, General Lagos 1163, Valdivia 5090000, Chile. FINANCIAMIENTO. Este trabajo fue apoyado y financiado por la Vicerrectoría de Investigación, Desarrollo y Creación Artística de la Universidad Austral de Chile, en el año 2017, a través del proyecto DID No S-2017-52.
} 
puntos, que posteriormente serán convertidos en una malla de polígonos para ser revestidas con una textura, obteniendo finalmente el modelo digital 3D (Arévalo Vera et al., 2015). El problema de esta práctica es el tiempo que se utiliza para poder obtener las imágenes y la dificultad que presenta para cubrir todos los ángulos de la estructura a digitalizar, con un ambiente de luz controlada, lo cual puede llevar varias horas dependiendo de la complejidad de la estructura a modelar. En nuestra experiencia, comenzamos con la fotogrametría con una cámara fotográfica Canon Reflex T2, donde obtuvimos modelos 3D de estructuras del laboratorio de anatomía humana de la Universidad Austral de Chile, algunas de buena calidad pero otras de calidad discutible, debido a la sensibilidad que presenta la obtención de varias fotografías de la muestra. No obstante, nos encontramos con el problema de cómo difundir o compartir estos modelos, para que funcionen como un recurso educativo abierto (REA) y estén disponibles a todos los usuarios que deseen utilizarlo, especialmente a estudiantes del área de la salud de las diferentes universidades del mundo. La creación de un software de tipo atlas anatómico suponía un gasto económico importante. Es por esto que el propósito del presente artículo, fue buscar la forma más económica y sencilla para crear una plataforma web que tenga las características de un atlas de anatomía, como REA con las estructuras digitalizadas en 3D del laboratorio de anatomía humana de la Universidad Austral de Chile, para que estudiantes desde cualquier rincón del mundo puedan acceder sin restricciones y de forma fácil y cómoda a través de su dispositivos electrónicos como computadores, celulares inteligentes o tablets.

\section{MATERIAL Y MÉTODO}

Para disminuir los tiempos de digitalización de las estructuras el año 2016 se postuló a un concurso interno de la Universidad Austral de Chile, a través de la Dirección de Investigación y Desarrollo (DID). Éste fue adjudicado el año 2017, él cual permitió la adquisición de un escáner 3D de marca Einscan, modelo Pro, que fue importado directamente desde China. Este equipo presenta cuatro modos de escaneo: escaneo rápido a mano, escaneo HD a mano, fijo sin placa giratoria y fijo con plataforma giratoria. Las dos primeras son para objetos de mayor tamaño, en tanto las últimas para muestras más pequeñas. El método que se utilizó fue la de escaneo fijo con plataforma giratoria (Fig. 1), debido a que presenta mejor definición, según el fabricante, en comparación a los demás métodos. Asimismo presenta la capacidad de escanear a color. La plataforma giratoria fue construida de madera y recubierta con una tela de color negra para evitar la refracción de la luz. La estructura de este equipo consta de dos cámaras fotográficas en cada extremo que toman fotos en blanco y negro y una cámara central que toma imágenes a color. La función es obtener gran cantidad de imágenes 2D en diferentes ángulos en poco tiempo. Este escáner cuenta con su propio software, cuyo propósito es agrupar las imágenes 2D obtenidas y convertirlas en modelos digitales 3D. La ventaja que presenta este sistema en relación a la fotogrametría digital, es la disminución significativa en cuanto a los tiempos de obtención de las imágenes y además agrega una mayor precisión en los detalles de éstas. En octubre del año 2017, con la colaboración de dos alumnos de pregrado de la carrera de kinesiología se eligieron piezas óseas para comenzar la digitalización, debido a su menor complejidad. Una vez dominado el sistema, se pasó a digitalizar otras estructuras como órganos, cortes de muestras anatómicas y articulaciones. Para la edición de los modelos se utilizó el software de código abierto Meshlab (Fig. 2), que permite ajustar la cantidad de polígonos del enmallado para reducir el peso del modelo. Además permite corregir algunas imperfecciones que se puedan haber suscitado durante el escaneo. Para subir, compartir y visualizar los modelos digitales 3D, se eligió la plataforma web Sketchfab, debido a su fluidez de navegación tanto en computadores, celulares inteligentes y tablets. Esta plataforma funciona como una red social que permite almacenar modelos digitales 3D en forma gratuita de hasta $50 \mathrm{mb}$ de peso con la posibilidad de etiquetar 5 estructuras en cada modelo. En nuestro caso, para poder etiquetar 20 estructuras de un modelo, se optó por la versión pro, el cual tuvo un costo de 15 dólares mensuales. Para que las muestras fueran de fácil acceso, se desarrolló una página web a través del sistema de gestor de contenidos (CMS) Wordpress, el que permite ordenar las muestras en los segmentos corporales correspondientes, siendo un sistema amigable para el usuario. Igualmente, permite otorgar acceso a otros tipos de contenidos educativos. En este mismo sentido, se adquirió un dominio sencillo y fácil de recordar: http://anatomiahumana3d.com.

\section{RESULTADOS}

Hasta el desarrollo de este artículo, se han digitalizado en modelos 3D y subidos a la plataforma de Sketchfab (Fig. 3), 76 muestras anatómicas del laboratorio de anatomía humana de la Universidad Austral de Chile, cuyas estructuras fueron debidamente etiquetadas utilizando los estándares de la terminología anatómica internacional (TAI). Estas muestras corresponden a: 60 muestras óseas, 3 muestras de cartílagos, 1 muestra de laringe, 7 muestras del sistema nervioso central, 2 muestras de órganos abdominales, 1 corazón, 1 muestra de genitales femeninos externos y 1 muestra de articulaciones. 


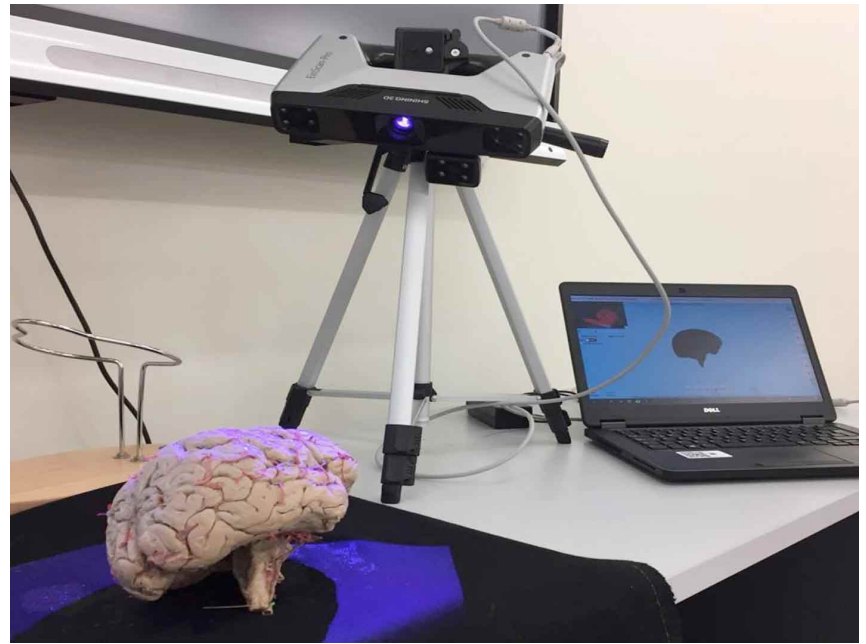

Fig. 1. Escáner 3D marca Einscan modelo Pro, como modo de escaneo fijo a través de un atril con plataforma giratoria donde se ubica la muestra, la plataforma se mueve de forma manual.

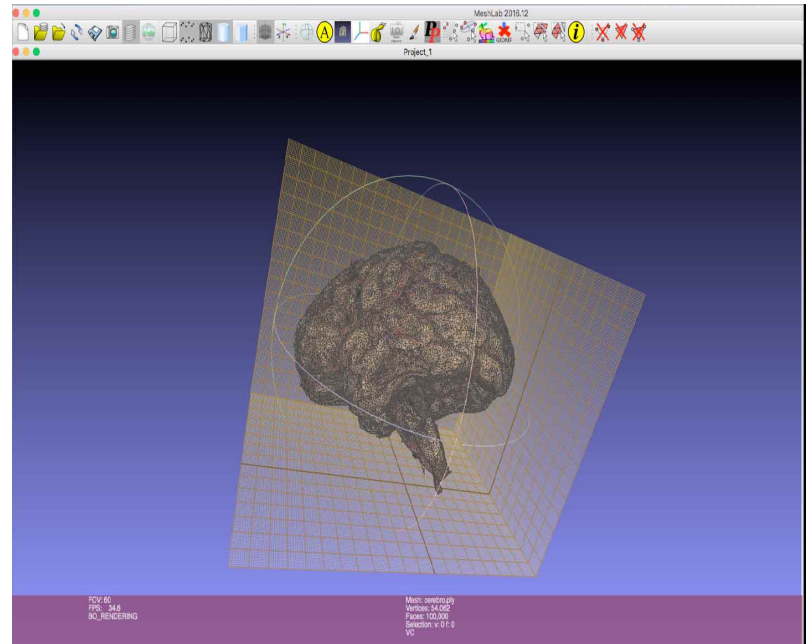

Fig. 2. Software Meshlab de código abierto, permite ajustar la cantidad de polígonos del enmallado para que el modelo digital no tenga un peso demasiado grande y pueda ser manejado fácilmente online. También permite arreglar algunas fallas del escaneo.

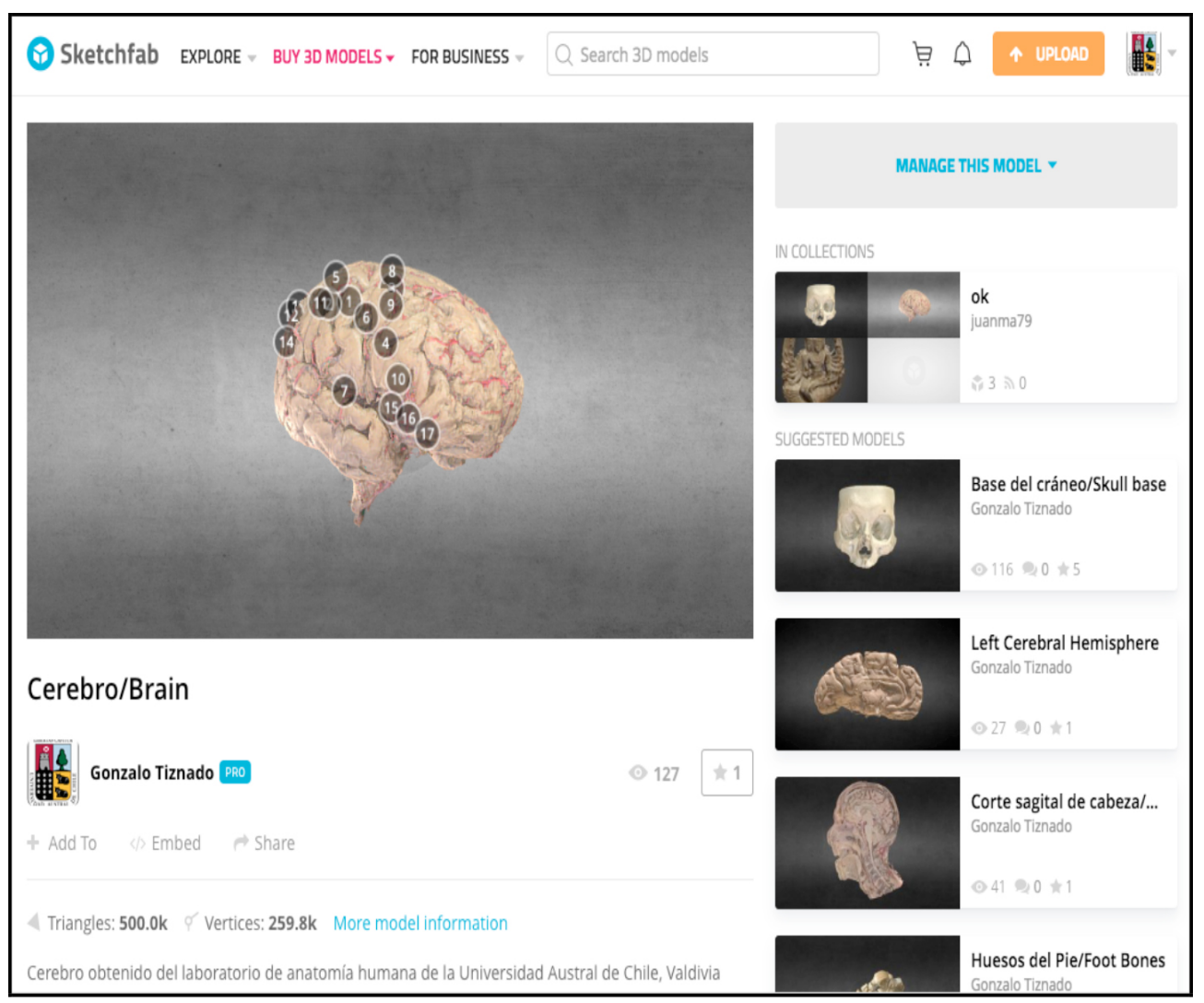

Fig. 3. Sketchfab, Plataforma web que permite subir modelos digitales 3D, el cuál además permite etiquetar las estructuras de un modelo.

La página web quedó alojada en el dominio http:// anatomiahumana3d.com (Fig. 4), abierto a cualquier persona que desee ingresar. Consta de un menú sencillo e intuitivo en español e inglés, que muestra datos de contacto, realizadores del proyecto, clases de anatomía y por último los modelos 3D. Al desplazar el cursor en clases de anatomía se encontrarán material educativo utilizado en las clases de las distintas carreras del área de la salud de la Universidad Austral de Chile, en formato de documento portátil (PDF), para que los alumnos puedan acceder al material antes de que 


\section{Wit Anatomía Humana $\exists D$ \\ $=$ Is Inicio Modelos $3 \mathrm{D}$ - Clases de anatomía Quiénes Somos Contáctanos \\ MODELOS 3D}

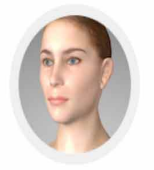

CABEZA Y CUELLO

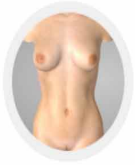

TRONCO

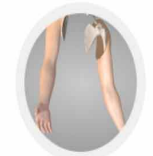

MIEMBRO SUPERIOR

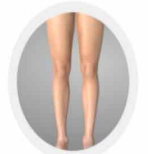

MIEMBRO INFERIOR
Fig. 4. Página web de dominio http://anatomiahumana3D.com, que posibilita organizar los modelos digitales $3 \mathrm{D}$ según el segmento corporal al que pertenece. Al hacer click sobre el segmento corporal seleccionado se despliegan los modelos, del segmento.

estas se presenten en la sala de clases. No obstante, estas clases pueden servir a otros estudiantes de cualquier universidad para contribuir a su aprendizaje. Al desplazar el cursor sobre Modelos 3D, se despliega un sub menú que muestra los segmentos corporales divididos en cabeza y cuello, tronco, miembro superior y miembro inferior. Esto permite seleccionar el modelo digital que desean observar. Al hacer click sobre la estructura digitalizada seleccionada, se redirige a una página donde se observa el modelo en cuestión, que no es más que la incorporación (embedded) del modelo alojado en Sketchfab. Aquí el usuario tendrá la opción de mover la muestra 3D en las diferentes direcciones observando las estructuras etiquetadas desde distintos ángulos y profundidades.

\section{DISCUSIÓN}

La creación de nuevos recursos educativos permite a los estudiantes tener más opciones al momento de estudiar. Vivimos en una época tecnológica donde los jóvenes necesitan mayor material interactivo para comprender los contenidos (García et al., 2014). Esto no es ajeno en anatomía, donde la falta de muestras cadavéricas y al aumento de alumnos año a año, hace que el tiempo de los prácticos sean restringidos. Frente a esta problemática se desarrolló esta plataforma que actúa como atlas de anatomía 3D online con muestras cadavéricas reales, el que no se necesita descargar y es completamente abierto a la comunidad. El estudiante una vez que haya pasado por el laboratorio puede llegar a su casa o desde cualquier lugar con conexión a internet e ingresar desde su computador, celular inteligente o tablets y repasar lo visto en el práctico.
Inzunza \& Bravo (1999) observaron el impacto que tienen los softwares de anatomía desarrollados por ellos mismos en la enseñanza de esta asignatura, especialmente con fotografías de preparados anatómicos de su propio laboratorio de anatomía y modelos esquemáticos. Por su parte, Guiraldes et al. (2001) también mostraron buenos resultados al complementar las clases prácticas y teóricas de anatomía con recursos educativos multimediales de fotografías que eran obtenidas del laboratorio de anatomía. Hasta ese momento los softwares de anatomía humana más conocidos eran el ADAM (Animated Dissection of Anatomy for Medicine) y el disco compacto de Netter que muestra esquemas interactivos de los sistemas anatómicos. Destaca también la creación de un software interactivo para la enseñanza del hígado por parte de Bucarey \& Álvarez (2006). Con el tiempo han aparecido softwares con esquemas interactivos en 3D y animaciones, como el Primal Pictures o el mismo ADAM que ha evolucionado hacia los modelos 3D. Sin embargo, los costos de estos programas hacen que su adquisición sea inalcanzable para un estudiante o un usuario común y corriente. La aparición de computadores más avanzados y a precios accesibles, ha permitido que más investigadores se acerquen al desarrollo de modelos $3 \mathrm{D}$, basados especialmente, en información entregada por las tomografías axiales computarizadas (TAC) (Pérez Gutiérrez \& Uribe Quevedo; Hu et al., 2009). No obstante, aún en el 2009 la tecnología era insuficiente para manejar modelos 3D de buena calidad, por ende los autores tenían que disminuir la cantidad de polígonos en el enmallado o utilizar computadores potentes que solo se encontraban en las instituciones universitarias. A partir ya del 2014 se ven varios artículos relacionados con el manejo de modelos 3D obtenidos de TAC o de resonancias nucleares magnéticas. A partir de estos sistemas autores han publicado, como la utilización de estos modelos pueden servir como material educativo, además de tener la posibilidad de imprimirlos en impresoras 3D, haciendo una replica casi exacta del modelo (Li et al., 2015; Inzunza et al., 2015; Pujol et al., 2016; Coronel et al., 2017; Lee \& Park, 2018). En una experiencia innovadora Inzunza et al. (2017) desarrollaron una plataforma de software educativo para optimizar la enseñanza de la anatomía, llamado Anatomicis Network, que consiste en la creación de organizaciones de instituciones universitarias que puedan administrar sus asignaturas de anatomía y mantenerlas comunicadas entre sí. En esta plataforma pueden comunicarse los académicos y estudiantes para acceder a material de estudio como textos, imagen 2D, Imagen 3D y videos. Los usuarios que pueden acceder a este contenido están restringidos a las organizaciones que participan. En este sentido nuestra experiencia que es mucho más limitada, busca que a través de plataformas sencillas, como una simple página web se puedan albergar recursos educativos abiertos, permitiendo a cualquier persona acceder a material de buena calidad. 
La finalidad de trabajar nuestros recursos como REA y permitir el acceso libre a cualquier usuario, va acorde con el llamado de la UNESCO (2002) en pro de la democratización de la educación. Permitiendo el acceso a estudiantes, de cualquier nivel educativo, que no tienen la posibilidad de acceder a recursos educativos con altos estándares de calidad, principalmente pedagógicos (Bucarey \& Aguilar, 2017). De otro lado, existe una ganancia para las instituciones que promueven el acceso abierto en su material científico y educativo, que es una mayor visibilidad e impacto de las instituciones a través de Internet (Babini et al, 2010).

Pretendemos en el futuro seguir agregando más contenido a la página, como videos, textos de corta duración y que tenga interacción con redes sociales. Por otro lado esperamos, igualmente, que a través de estos modelos, instituciones o personas que posean impresoras 3D puedan descargar estos archivos e imprimirlos para realizar trabajos, como métodos de enseñanza activa. Nuestro objetivo final es sumar más instituciones universitarias, que permitan agregar más contenido de modelos 3D de muestras anatómicas reales, que puedan ayudar al mejoramiento de la comprensión de la anatomía por parte de los estudiantes.

TIZNADO-MATZNER, G.; BUCAREY-ARRIAGADA, S. \& LIZAMA, P. R. Experience in the creation of an online platform to host three-dimensional models of real anatomical pieces to be shared as open educational resources (OER). Int. J. Morphol., 37(4):1267-1271, 2019.

SUMMARY: For a long time, the teaching of human anatomy, apart from dissections and observations of cadaveric samples, has been based on texts and later complemented with softwares with two-dimensional (2D) images or models that imitated anatomical structures. With the development of technology in recent years, three-dimensional (3D) digital modeling has been booming, to which anatomy has not been oblivious. Through digital photogrammetry this objective can be achieved, since it is a method that consists of capturing a large number of images from the different angles of an object, in a controlled light environment, and then to be entered into specialized software, which is responsible for grouping and generating the necessary information so that the volume and depths of the object are converted into a mesh of polygons, to be subsequently coated with a texture, thus obtaining a 3D digital model. The problem with this method is the time it takes to take good quality images. For this reason, through the Directorate of Research and Development of the Universidad Austral de Chile, a 3D scanner was acquired, a device that allows obtaining a large number of images at different angles in less time, and through its own software creates 3D digital models. We have digitized 76 anatomical samples from our human anatomy laboratory, which were uploaded to the web platform Sketchfab, a social network that specializes in sharing 3D digital models. After this an open web page was created that allows organizing the models hosted in Sketchfab, according to the body segment to which they belong. This page can be accessed by anyone, from any place that has internet, through a personal computer, smart cell phone or tablets, making it an open educational resource (OER).

KEY WORDS: 3D Digitals Models; Anatomical Samples; Open Educational Resource; Web Platform.

\section{REFERENCIAS BIBLIOGRÁFICAS}

Arévalo Vera, B.; Bayona Ibáñez, E. \& Rincón Parada, I. K. Metodología para documentación 3D utilizando fotogrametría digital. Tecnura, 19:113-20, 2015.

Babini, D.; González, J.; López, F. \& Medici, F. Social construction of institutional repositories: the case of a Latin America and the Caribbean repository. Inf. Cult. Soc., (23):63-90, 2010.

Bucarey, S. \& Álvarez, L. Methodology to construct learning object for teaching human anatomy in integrated courses. Int. J. Morphol., 24(3):357-62, 2006.

Bucarey, S. G. \& Aguilar, M. L. Recursos educativos abiertos en la Facultad de Medicina de la Universidad Austral de Chile, proyecto AUS1410. Form. Univ., 10(2):23-30, 2017.

Coronel, J.; Palacio, J. \& Rueda-Esteban, R. Multiple software based 3D modeling protocol for printing anatomical structures. Int. J. Morphol., 35(2):425-9, 2017.

García, T. J. A. G.; Avendaño, P. R. \& Martínez, A. J. J. The use of technology in the teaching of anatomy in Mexico and its comparison with international education. Rev. Fac. Med. U. N. A. M., 57(3):31-9, 2014.

Guiraldes, H.; Oddó, H.; Mena, B.; Velasco. N. \& Paulos, J. Teaching human anatomy: experience and challenge in a medical school. Rev. Chil. Anat., 19(2):205-12, 2001.

Hu, A; Wilson, T; Ladak, H; Haase, P. \& Fung, K. Three-dimensional educational computer model of the larynx: voicing a new direction. Arch. Otolaryngol. Head Neck Surg., 135(7):677-81, 2009.

Hunter, A. ADAM Interactive Anatomy. The Anatomy Project. B. M. J., 320:521, 2000 .

Inzunza, O. \& Bravo, H. Impact of two software of human anatomy in the performance of the students practical knowledge. Rev. Chil. Anat., 17(2):2059, 1999.

Inzunza, O.; Caro, I.; Mondragón, G.; Baeza, F.; Burdiles, A. \& Salgado, G. 3D impressions, new technology that supports anatomical teaching. Int. J. Morphol., 33(3):1176-82, 2015.

Inzunza, O.; Neyem, A.; Sanz, M. E.; Valdivia, I.; Villarroel, M.; Farfán, E.; Matte, A. \& López-Juri, P. Anatomicis Network: a cloud-based educational software platformto enhance anatomy teaching in medical education. Int. J. Morphol., 35(3):1168-77, 2017.

Lee, S. E. \& Park, J. S. Automatic 3D modeling of liver segments including segmental branches of portal triad and hepatic vein based on the sectionedimages. Int. J. Morphol., 36(2):402-6, 2018.

Li, Z.; Li, Z.; Xu, R.; Li, M.; Li, J.; Liu, Y.; Sui, D.; Zhang, W. \& Chen, Z. Three-dimensional printing models improve understanding of spinal fracture--A randomized controlled study in China. Sci. Rep., 5:11570, 2015.

Pérez Gutiérrez, B. A. \& Uribe Quevedo, A. J. A multimedial tool to study the ear anatomy from virtual models. Cienc. Ing. Neogranad., 19(2):29-43, 2009.

Petriceks, A. H.; Peterson, A. S.; Angeles, M.; Brown, W. P. \& Srivastava, S. Photogrammetry of human specimens: an innovation in anatomy education. J. Med. Educ. Curric. Dev., 5:2382120518799356, 2018.

UNESCO. Forum on the Impact of Open Courseware for Higher Education in Developing Countries. Paris, UNESCO, 2002. Disponible en: https:// unesdoc.unesco.org/ark:/48223/pf0000128515.

\section{Dirección para correspondencia: \\ Gonzalo Tiznado Matzner \\ Instituto Anatomía, Histología y Patología \\ Universidad Austral de Chile. \\ Campus Isla Teja S/N \\ Valdivia \\ CHILE}

Email: gonzalo.tiznado@uach.cl

Recibido : 10-04-2019

Aceptado: 21-06-2019 